\title{
Circuit
}

Musiques contemporaines

Cage/Satie, le silence vertigineux

Quelques rencontres avec John Cage sans jamais l'avoir

regardé dans les yeux

Cage/Satie, the dizzying silence

Meetings with John Cage without ever having looked him in the

eye

\section{Rober Racine}

Volume 8, numéro 2, 1997

Québecage

URI : https://id.erudit.org/iderudit/902204ar

DOI : https://doi.org/10.7202/902204ar

Aller au sommaire du numéro

Éditeur(s)

Les Presses de l'Université de Montréal

ISSN

1183-1693 (imprimé)

1488-9692 (numérique)

Découvrir la revue

Citer cet article

Racine, R. (1997). Cage/Satie, le silence vertigineux : quelques rencontres avec John Cage sans jamais l'avoir regardé dans les yeux. Circuit, 8(2), 39-44.

https://doi.org/10.7202/902204ar
Résumé de l'article

L'auteur raconte comment, sans avoir eu l'occasion de rencontrer Cage, il a été mis en contact avec lui par l'intermédiaire de Satie dont il a joué les Vexations à quatre reprises. Il évoque également son oeuvre visuelle, La musique d'une Cage. 


\section{Cage/Satie : le silence vertigineux Quelques rencontres avec John Cage sans jamais l'avoir regardé dans les yeux Rober Racine}

"Moi seul suis humain, tout le reste divin » L'Innommable, Samuel Beckett.

C'est la nuit. J'observe la comète Hale-Bopp en écoutant Four ${ }^{3}$ (1991) de John Cage ("). Une œuvre pour deux pianos, violon et douze « bâtons de pluie » (rainsticks) basée sur le thème des Vexations d'Erik Satie.

Entre ces deux événements, la comète et Four ${ }^{3}$, il y a correspondance, réponse, écho de trajectoires. Une image commune les relie pour moi : l'immobilité dans ce qu'elle a de plus vertigineux. Paradoxe du mystère.

La comète file à une vitesse folle, à des milliers de kilomètres de nous, de notre centre. Elle effleure à peine notre vision. II y a là-haut un point fixe, une douce lueur qui scintille au nord-ouest. Le phénomène reviendra dans quelques dizaines d'années. Tout près de la répétition, récurrence astronomique, la comète est un thème qu'on reconnaît, devine. II offre de multiples variations de tempi, de couleurs, de motifs. C'est une vitesse qui se consume pour nous faire rêver.

Four $^{3}$ me rappelle cette rencontre entre un thème, Vexations (1893-1895) pour piano d'Erik Satie, et l'auditeur observateur. La comète Vexations revient presque cent ans plus tard sous la forme de Four ${ }^{3}$, une autre forme, quelque peu altérée, mais dont le vacillement demeure.

Comme Vexations, la comète Hale-Bopp ef Four $^{3}$ nous offrent une expérience de contemplation et de méditation, dans le calme et le silence de la nuit.

Dans la nuit du 10 avril 1977, je rencontre pour la première fois le duo Cage-Satie. C'est par l'entremise des mots que cela se produit : le livre d'Anne Rey (1974) sur Erik Satie. Je dis «les mots» et non les sons puisque ce sont eux qui m'ont introduit à la pensée des deux compositeurs. A la page 171, l'auteure cite un texte de Cage tiré de Silence (1961, p. 78) à propos de
(1) Cage, John, «The Number Pieces 1 ", The Complete John Cage Edition, volume 12, Radio-France/Mode Records, Mode 44, 1995. 
Satie, plus spécifiquement des Vexations: "[...] Certes, on ne pourrait pas supporter une exécution de Vexations /qui dure, selon moi, vingt-quatre heures: 840 répétitions d'un morceau de 52 mesures à structure répétitive : $A, A_{1} ; A$, $A_{2}$, chaque $A$ ayant 13 mesures de longl, mais pourquoi y songer? " Ce texte me provoqua. J'écrivis en bas de page : «Faut y songer et surtout jouer cette pièce. La musique doit être entendue et écoutée; non pas lue. " Sans le savoir, Cage venait de me lancer un défi.

Cage a publié son texte en 1958. C'est en 1963, au Pocket Theatre à New York, qu'il exécuta Vexations avec un groupe de pianistes; puis en 1966 pour une durée de 18 heures 40 minutes $(1 \times 840$ ?...).

Pour ma part, j'ai exécuté à quatre reprises, en solo, les Vexations: le 4 novembre 1978 à Montréal en 14 heures 8 minutes; à Arthabaska le 15 décembre 1978 en 17 heures 59 minutes; à Toronto le 13 janvier 1979 en 19 heures; enfin le 19 mai 1979 à Vancouver en 17 heures ${ }^{(2)}$.

Aujourd'hui, je me dis que le texte de Cage et son fameux "pourquoi y songer " m'ont ouvert la porte d'un monde insoupçonné.

Pas plus que la comète Hale-Bopp, je n'ai jamais rencontré John Cage. Je l'ai effleuré quelques fois. Ce fut d'abord par le livre d'Anne Rey sur Satie. Je me souviens de ma fascination devant le dessin du schéma formel pour Cartridge Music (page 172). Je me suis dit à ce moment-là : j'aimerais écrire de la musique comme cela. Déjà le paradoxe de l'écriture... Puis ce fut la lecture de ses textes regroupés dans Silence; enfin les merveilleux entretiens avec Daniel Charles: Pour les oiseaux (Cage, 1976). Ma première impression musicale avec le monde de Cage fut à la radio par l'audition du Quatuor à cordes et quelques pièces pour piano préparé qui m'ont envoûté. J'avais à peine vingt ans.

Au début de janvier 1979, j'exécutai pour la troisième fois, en solo, les Vexations à la Music Gallery à Toronto. John Cage était de passage à Montréal. C'est le regretté Claude Vivier qui m'apprit sa venue. Claude m'a dit qu'il avait discuté de virtuosité avec Cage. L'idée intéressait Cage parce qu'elle lui permettait de "réaliser l'impossible». J'écoutais le récit de Vivier et je me disais : la vie est étrange. Pendant que je joue Vexations à Toronto, Cage est à Montréal.

J'aurais aimé le rencontrer pour lui poser une seule question : comment s'y était-il pris pour compter les 840 motifs de Vexations? C'est tout. Une demande "technique ", diront certains, mais incontournable pour qui veut exécuter Vexations intégralement. Comment capter le pouls d'une œuvre, suivre les battements de cœur d'une nuit, d'une vie?

Personnellement, ma solution fut très simple. Sur une toute petite boîte $120 \times$ $20 \mathrm{~cm}$ ) j'ai enfoncé, au préalable et à mi-hauteur, 840 épingles que j'abaissais complètement après exécution d'un motif lors du concert. En alignant vingt
(2) Pour le récit de ces exécutions, je renvoie le lecteur à mon texte « Vexations»(Racine, 1979, pp. 50-53). 
et une rangées de quarante épingles, j'obtenais ainsi une idée générale, mais jamais précise, du compte. Cette boîte est placée sur le piano, juste au-dessus du clavier. Après avoir joué plusieurs motifs, l'action d'enfoncer l'épingle correspondante fait partie intégrante de la gestuelle pianistique de l'œuvre. C'est pour moi la façon idéale de compter les 840 motifs de Vexations pour une exécution solo. Un clavier à épingles, donc, silencieux en réponse peutêtre au piano préparé de Cage. Avec ce dispositif, j'ai placé sur le piano une boîte à silence, une façon d'accompagner et de noter le déroulement de l'œuvre. Par le piano préparé, Cage a aussi trouvé une façon, toute simple, de modifier la nature d'un objet (piano) pour en révéler une dimension nouvelle, différente. II ne faut pas oublier que dans les deux cas, il s'agissait de trouver une solution à un problème précis; sans plus ${ }^{(3)}$.

De Vexations au "silence " de 4'33", il y a plus d'une similitude. La partition de Vexations se termine par un silence ; celle de 4'33" par le son : celui de la vie. C'est le complément parfait. II s'agit d'une coïncidence poétique, d'une rencontre idéale. Imaginez une version de $4{ }^{\prime} 33^{\prime \prime}$ pour piano préparé ; ou Vexations encore...

En écoutant Four ${ }^{3}$ où le thème des Vexations est présenté, taillé, modelé par le silence de façon aussi sensible, je ne peux m'empêcher de penser qu'il y a chez Cage la réalisation d'un idéal musical entrevu par Satie. En cela, la musique de Cage est une merveilleuse écoute du monde spirituel de Satie.

A qui sait écouter ces silences, une grande leçon d'humilité est offerte ici.

Hale-Bopp est légèrement à gauche maintenant, un peu plus au nord-ouest. Comme si la comète effectuait un déplacement stéréophonique.

Cela me rappelle la voix et le grand rire d'enfant de John Cage. La première fois où j'ai entendu son rire et sa voix ce fut à l'audition d'un documentaire radiophonique réalisé par Glenn Gould sur Arnold Schœnberg. C'était en 1984 et je préparais pour la FM de Radio-Canada un documentaire sur Gould. Dans le documentaire sur Schœnberg, Cage évoque sa rencontre avec l'auteur du Pierrot lunaire en terminant son témoignage par un éclat de rire. Tellement communicatif que Gould essaie de retenir son propre rire durant l'entretien. Pour moi, la beauté de ce passage radiophonique tient à ceci. Au montage, Gould a diffusé ce rire en le faisant passer du haut-parleur droit au haut-parleur gauche, doucement. Comme si la voix et le rire de Cage étaient un arc-en-ciel sonore au-dessus de l'esprit de Schœenberg. Mystérieuse rencontre: Schœnberg, Gould et Cage. Du silence de l'Europe aux rires de l'Amérique, notre écoute et celle du monde.

Là où j'ai éprouvé une grande émotion avec John Cage, peut-être la plus forte, c'est en l'écoutant lire les mots du mesostic Sculpture Musicale (1985(4)). La voix d'un être humain est unique et le résume parfois tout entier. Celle de
(3) Dans mon cas, il s'agissait de trouver une façon simple et discrète de compter les 840 motifs de l'œuvre sans l'aide d'une autre personne. Dans le cas de Cage, le piano préparé fut « inventé » pour remplacer un orchestre d'instruments de percussion impossible à loger dans un théâtre pour accompagner le ballet Bacchanale de Syvilla Fort en 1939. Cf. Cage (1976), pp. 29-30.

(4) « Music by Marcel Duchamp », DDD/Éditions Block, EB 202, 1991. 
Cage lisant sa poésie reflète celle de l'enfance. Ce temps de l'innocence où il n'y a jamais de tension dans le regard, juste une interrogation, une quête ; aucune prétention. Comme si le don offert était l'âme de l'attente. La fragilité de sa voix, peut-être parce qu'il parle français, peutêtre parce qu'il s'agit de mots tout simples, rejoint le «presque rien » du tissu sonore de Four ${ }^{3}$. C'est ce qui fait sa force et sa densité. Comme la petite lueur de la comète Hale-Bopp dans le ciel, ces quelques traînées de sons transportent avec elles la poésie de ce qui naît.

Comme Satie, Cage a joué du piano, composé de la musique, collaboré avec des danseurs, fréquenté le milieu des arts visuels, lu des conférences, écrit et publié plusieurs articles, exploré les nouvelles technologies de son époque, communiqué son humour. $\|$ a dessiné également.

En 1989, John Cage est venu à Montréal et a exposé à la galerie Graff plusieurs de ses dessins. Ce fut une autre occasion où j'aurais pu le rencontrer et réaliser une entrevue pour la FM de Radio-Canada. C'est mon collègue d'alors, le critique d'art Gilles Daignault, qui eut ce plaisir. Par un pur hasard, autre coïncidence poétique, le jour où i'aurais pu réaliser cet entretien, i'avais l'opportunité d'aller à Cap-Tourmente observer et entendre le spectacle fascinant des grandes oies blanches. J'avoue avoir hésité entre rencontrer John Cage ou vivre cette expérience dans Charlevoix. Puis, je me suis dit que Cage aurait fait le voyage à Cap-Tourmente. II m'aurait sûrement dit : il y a là-bas autant, sinon plus, à apprendre.

Aujourd'hui, je souris en me disant que j'ai contourné Cage... pour les oiseaux.

En 1993, j'ai été invité à présenter une œuvre visuelle pour l'exposition "Not wanting to say anything about John » Hommage à John Cage. L'exposition s'est tenue à l'École des beaux-arts de Roven en France et réunissait des œuvres multimédias de 35 artistes internationaux ${ }^{(5)}$.

Pour l'occasion, j'ai réalisé la musique d'une Cage, c'est-à-dire la PageMiroir No 234 contenant le mot «cage». Cette page, qui est extraite du dictionnaire Le Petit Robert, édition 1979, est enluminée et déposée sur un miroir. Il y a sur la page, à l'intérieur et autour des mots, plusieurs types de notations: graphique, linguistique et musicale. Pour cette dernière, j'ai extrait de la définition du mot « cage " le nom des notes de la gamme que l'on retrouve dans plusieurs mots de la langue française (résolution, famille, faut, silence, etc.). J'ai souligné ces notes telles qu'elles apparaissent dans la définition: de haut en bas, de gauche à droite. Puis j'ai simplement reporté le tout sur une portée. Comme toujours dans ce cas, la consigne de jeu est minimale : un mot $=$ un temps. S'il y a une seule note dans un mot (silence), cela donne une noire; deux notes (famille), deux croches, etc. Cette œuvre correspond bien à l'esprit ludique de Cage. Pour lui, le mot, l'objet, l'image, la matière, le mouvement, le son ou tout autre élément peut révéler une musique secrète, cachée.
(5) II s'agit, dans l'ordre alphabétique, de Laurie Anderson, Michel Aubry, Dominique Bailly, Gavin Bryars, Jean-Paul Berrenger, Mark Brusse, John Cage, John Cale, Daniel Charles, Brian Eno, Esther Ferrer, Robert Filliou, Richard Hamilton, Dick Higgins, Tom Johnson, Soun-Gui Kim, Alison Knowles, Richard Kostelanetz, Jean-Jacques Lebel, Joan Logue, Charlotte Moorman, Max Neuhaus, Yoko Ono, Nam June Paik, Philippe Parreno, Rober Racine, David Revill, Erik Samakh, Carolee Schneemann, José-Maria Sicillia, David Tudor, Greg Ulmer, Ben Vautier, Eric De Visscher, Huang Yong Ping. L'exposition, organisée par Jean-Jacques Lebel, s'est tenue du 3 au 30 juin 1993. 
Four ${ }^{3}$ se termine doucement. Elle laisse dans ma mémoire un calme bienfaisant. Hale-Bopp poursuit une route que ma rétine ne capte plus maintenant. Chaque œuvre possède sa nuit et son jour. Chaque œuvre offre un spectre infini de nuances et de possibilités. À la fois fugitive et prisonnière de sa propre forme, la musique transcende notre écoute pour révéler une face cachée de nous-mêmes. Dans ses entretiens avec Daniel Charles, Cage définit le rythme comme "quelque chose d'inattendu, d'irrelevant " (Cage, 1976, p. 224). II faut être disponible, saisir l'occasion, suivre une attirance, même si elle nous semble incompréhensible sur le moment.

Satie m'a présenté Cage qui à son tour m'a fait découvrir le monde vertigineux des Vexations. L'un et l'autre sont pour moi l'exemple parfait de l'artiste qui s'interroge face à l'art et la création, face au monde dans lequel il évolue. Tous les deux ont une faculté d'émerveillement et de questionnement unique. Elle s'exprime dans leurs écrits, compositions musicales, dessins, conférences, actions sur leurs contemporains. II y a plus: ils proposent chacun à leur manière une attitude face à la vie et nous invitent à prendre le temps. L'Histoire retiendra peut-être de Cage ce qu'elle a retenu de Satie. Je ne sais pas.

Erik Satie a correspondu avec Claude Debussy comme John Cage avec Pierre Boulez. Une amitie et un respect mutuels existaient entre eux. Certes, il y aurait un texte fascinant à écrire sur ces quatre musiciens, mais pourquoi y songer ?...

CAGE, J. (1961), Silence, Middletown, Wesleyan University Press.

CAGE, J. (1976), Pour les oiseaux, entretiens avec Daniel Charles, Paris, éditions Pierre Belfond.

RACINE, R. (1979), "Vexations », Parachute, n 15, été, pp. 50-53.

REY, A. (1974), Erik Satie, Paris, Seuil. 


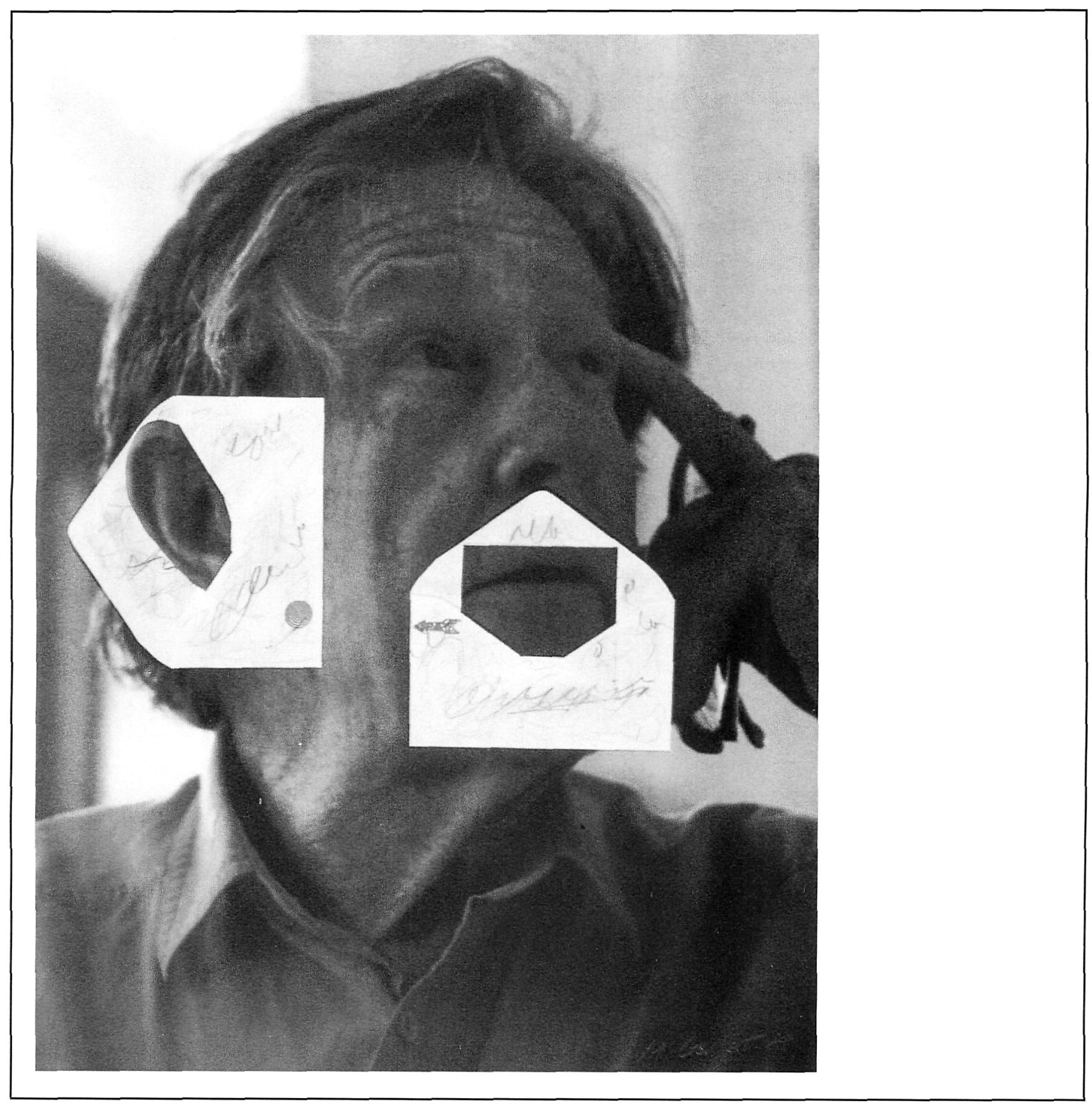

